\title{
Salmonella Prostatic Abscess in an Immunocompetent Patient
}

\author{
Christina Lin $^{1}$, Satya Patel ${ }^{1}$ \\ 1. Internal Medicine, University of California Los Angeles, Los Angeles, USA
}

Corresponding author: Christina Lin, cqlin@mednet.ucla.edu

\begin{abstract}
Patients returning from travel to a foreign country often present with atypical infections that can present as diagnostic challenges. Although rarely seen in the United States, Salmonella infections are commonly seen in Sub-Saharan Africa. The common clinical manifestations of Salmonella infection include fever and diarrhea; however, about $5 \%$ of cases of non-typhi Salmonella progress to bacteremia. Here, we present a case of a unique presentation of Salmonella infection manifesting as a prostatic abscess in an immunocompetent patient.
\end{abstract}

Categories: Internal Medicine, Infectious Disease

Keywords: salmonella, fever, bacteremia, prostatic abscess, travel

\section{Introduction}

Non-typhoidal salmonellosis is a common cause of diarrhea worldwide though occurs most frequently in Sub-Saharan Africa, where 79\% of all cases globally are found [1]. Other endemic regions include South Asia, Latin America, and the Middle East. Risk factors for disease include an immunocompromised state, malaria, hemoglobinopathies (including sickle cell trait), chronic liver disease, and chronic granulomatous disease. Non-typhoidal Salmonella are associated with ingestion of poultry, milk products, eggs, fresh produce, and contact with animals. Common features of infection include fever, abdominal pain, and diarrhea. Most infections are self-limited; however, up to $5 \%$ of cases result in bacteremia and extraintestinal manifestations. Risk factors for more invasive disease include infants ( $<12$ months) or older age ( $>50$ years), $\mathrm{HIV}$, or any other immunocompromising condition. Invasive disease is seen most commonly in nontyphoidal Salmonella serovars that are endemic to Sub-Saharan Africa [2-4].

\section{Case Presentation}

A 50-year-old immunocompetent male with past medical history significant for childhood malaria presented with fevers and myalgias following a trip to Ghana one week prior. He initially denied any focal infectious symptoms, including cough, chest pain, shortness of breath, dysuria, nausea, abdominal pain, or diarrhea. During the trip, he stayed in a rural village with indoor plumbing, ate home-cooked food, drank bottled water, and was not aware of any sick contacts.

Received 06/08/2020

an $07 / 04 / 2020$ Review ended 07/05/2020 Published 07/10/2020

\section{() Copyright 2020}

Lin et al. This is an open access article distributed under the terms of the Creative Commons Attribution License CC-BY 4.0., which permits unrestricted use, distribution, and reproduction in any medium, provided the original author and source are credited.
His presenting vital signs were as follows: temperature $102^{\circ} \mathrm{F}$, heart rate $133 \mathrm{bpm}$, blood pressure $127 / 88$ $\mathrm{mmHg}$, and oxygen saturation $98 \%$ on ambient air. On physical exam, he appeared fatigued with dry mucous membranes, was tachycardic with a regular rhythm, but otherwise had no significant findings. His laboratory testing showed a white blood cell count of $12,000 / \mu \mathrm{L}$ with $86.1 \%$ neutrophils and 70 bands $/ \mu \mathrm{L}$. Initial blood cultures, urinalysis, and chest X-ray were unremarkable. Due to his recent travel and concern for infection, a broad infectious work-up was done. Nasal influenza/respiratory syncytial viral swab, HIV1/HIV-2, dengue IgM, Zika IgM, and blood smears for malaria were negative. Given his persistent high fevers and recent travel to an endemic area for malaria, he was empirically treated for malaria with atovaquone/proguanil without significant improvement in his symptoms. On hospital day 2, the patient had a temperature to $101.6^{\circ} \mathrm{F}$ and developed dysuria and gross hematuria. A repeat urinalysis demonstrated pyuria and $2+$ blood, and he was empirically started on ceftriaxone.

His urine culture later revealed ceftriaxone-sensitive Salmonella non-typhi Group B serotype. The patient continued to have fevers and on hospital day 4, the patient developed non-bloody diarrhea. Stool bacterial testing demonstrated the same strain of Salmonella. Blood cultures continued to be negative. Due to his persistent fevers, a CT scan of his abdomen and pelvis with intravenous contrast was obtained and showed multifocal prostatic abscesses (Figure 1). The patient underwent transgluteal drainage of his prostatic abscesses and the resultant cultures grew the same pathogen. His fever curve subsequently improved, and he was discharged on an extended course of trimethoprim-sulfamethoxazole. 


\section{Cureus}

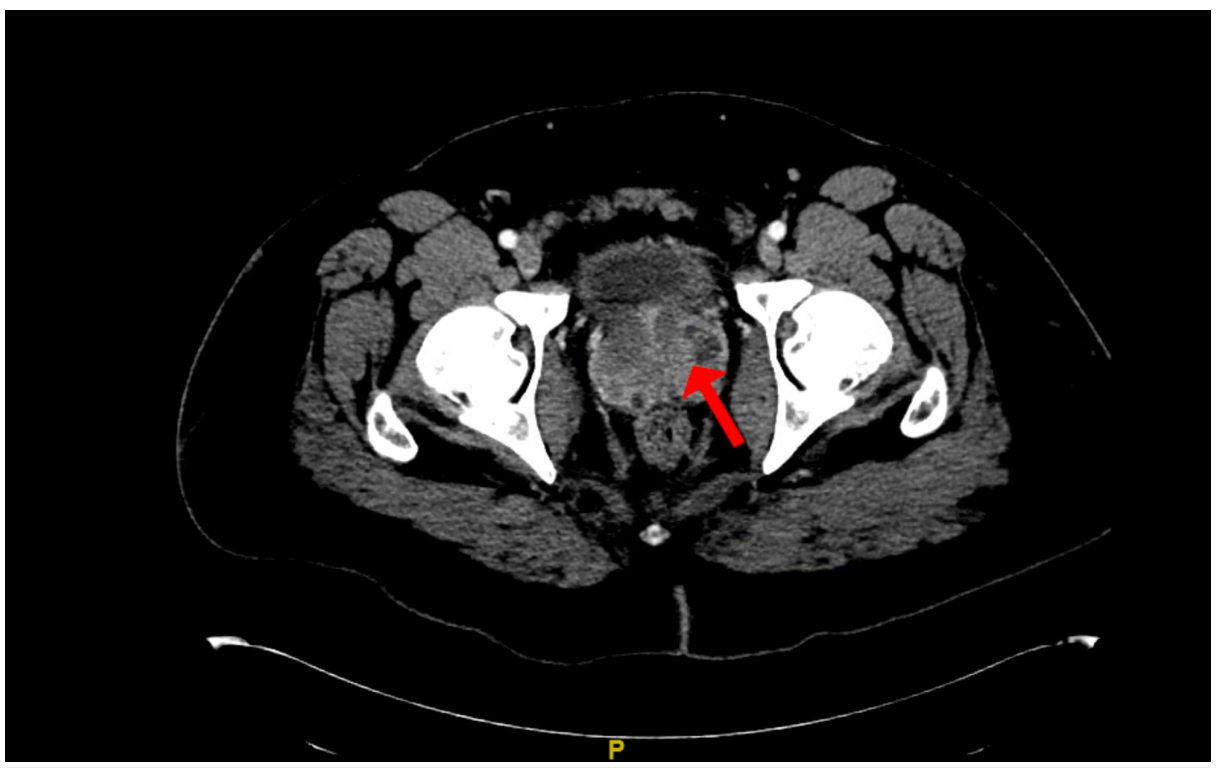

FIGURE 1: CT of the abdomen/pelvis with contrast demonstrating a moderately enlarged, heterogeneous appearing prostate gland with multiple hypoattenuating foci within it and surrounding inflammatory change concerning for multifocal prostatic abscesses.

\section{Discussion}

The most common manifestation of Salmonella infection is gastroenteritis. The pathogenesis of infection involves ingestion of the bacteria into the gastrointestinal tract, whereby the microorganism must survive in the acidic environment of the stomach, and eventual adherence and invasion of the M cells within the Peyer patches of the colon. Alterations of the gastrointestinal tract, such as acid-reducing medications or antibiotic use, can increase the risk of progression from enteric to systemic salmonellosis [5]. Bacteremia and disseminated infection is estimated to occur in about $5 \%$ of cases, most commonly with the higher virulence strains of non-typhi Salmonella [6]. Salmonella bacteremia can result in infection at almost any site, the most common sites of dissemination being urinary, lung, bones, and central nervous system. A study in Malaysia found that immunocompromised patients with Salmonella infection not only had a higher mortality but also were more likely to present with primary bacteremia compared to their immunocompetent counterparts [7]. Thus, a patient with disseminated salmonellosis should be worked up for any underlying immunocompromising condition. Uncomplicated disease can be treated with supportive care without antibiotics; however, those with extraintestinal complications require a prolonged antibiotic course and often surgical interventions.

This case represents a unique presentation of Salmonella infection manifesting as a prostatic abscess. Prostatic abscesses due to Salmonella are exceedingly rare and have previously only been described in immunocompromised patients, which was not the case in our patient [8]. Most cases of extraintestinal salmonellosis are a result of hematological spread from gut translocation $[9,10]$. Although it is unclear whether the primary source of infection in this case was gut translocation or ascending urinary tract infection, spread between the sites was most likely hematologic as bacteremia is a known complication of Salmonella infections. This raises a diagnostic dilemma as to how to interpret the negative blood cultures. The sensitivity for non-typhoid Salmonella on blood cultures is unknown, although it is notable that the sensitivity of typhoid Salmonella on blood cultures is only 50\%-70\% [11]. It is therefore of high utility to obtain a detailed clinical exam to assess for evidence of disseminated salmonellosis even in the presence of negative blood cultures.

\section{Conclusions}

A patient traveling from endemic areas of Salmonella infection such as Africa should raise clinical suspicion for salmonellosis. Mortality and morbidity from disseminated Salmonella infections are high. Clinicians should be aware of the extraintestinal complications of disseminated Salmonella infections, which can often serve as diagnostic challenges.

\section{Additional Information}

\section{Disclosures}


Human subjects: Consent was obtained by all participants in this study. Conflicts of interest: In compliance with the ICMJE uniform disclosure form, all authors declare the following: Payment/services info: All authors have declared that no financial support was received from any organization for the submitted work. Financial relationships: All authors have declared that they have no financial relationships at present or within the previous three years with any organizations that might have an interest in the submitted work. Other relationships: All authors have declared that there are no other relationships or activities that could appear to have influenced the submitted work.

\section{References}

1. Majowicz SE, Musto J, Scallan E, et al.: The global burden of nontyphoidal Salmonella gastroenteritis . Clin Infect Dis. 2010, 50:882-889.

2. Kingsley RA, Msefula CL, Thomson NR, et al.: Epidemic multiple drug resistant Salmonella Typhimurium causing invasive disease in sub-Saharan Africa have a distinct genotype. Genome Res. 2009, 19:2279-2287.

3. Keddy KH, Musekiwa A, Sooka A, et al.: Clinical and microbiological features of invasive nontyphoidal Salmonella associated With HIV-infected patients, Gauteng Province, South Africa. Medicine. 2017, 96:e6448.

4. Akullian A, Montgomery JM, John-Stewart G, et al.: Multi-drug resistant non-typhoidal Salmonella associated with invasive disease in Western Kenya. PLoS Negl Trop Dis. 2018, 12:e0006156.

5. Hung TY, Liu MC, Hsu CF, Lin YC: Rotavirus infection increases the risk of bacteremia in children with nontyphoid Salmonella gastroenteritis. Eur J Clin Microbiol Infect Dis. 2009, 28:425-428. 10.1007/s10096008-0641-0

6. Saphra I, Winter JW: Clinical manifestations of salmonellosis in man; an evaluation of 7779 human infections identified at the New York Salmonella Center. N Engl J Med. 1957, 256:1128-1134. 10.1056/NEJM195706132562402

7. Dhanoa A, Fatt QK: Non-typhoidal Salmonella bacteraemia: epidemiology, clinical characteristics and its' association with severe immunosuppression. Ann Clin Microbiol Antimicrob. 2009, 8:15. 10.1186/14760711-8-15

8. Giessing M, Baumgart E, Lenk S, Loening SA: Salmonella Typhimurium prostatic abscess: a rare cause of persisting dysuria in an AIDS patient. AIDS. 2003, 17:449-450.

9. Huang DB, DuPont HL: Problem pathogens: extra-intestinal complications of Salmonella Enterica serotype Typhi infection. Lancet Infect Dis. 2005, 5:341-348.

10. Eng SK, Pusparajah P, Ab Mutalib NS, Ser HL, Chan KG, Lee LH: Salmonella: a review on pathogenesis, epidemiology and antibiotic resistance. Front Life Sci. 2015, 8:284-293. 10.1080/21553769.2015.1051243

11. Mogasale V, Ramani E, Mogasale VV, Park J: What proportion of Salmonella Typhi cases are detected by blood culture? A systematic literature review. Ann Clin Microbiol Antimicrob. 2016, 15:32. 10.1186/s12941016-0147-z 